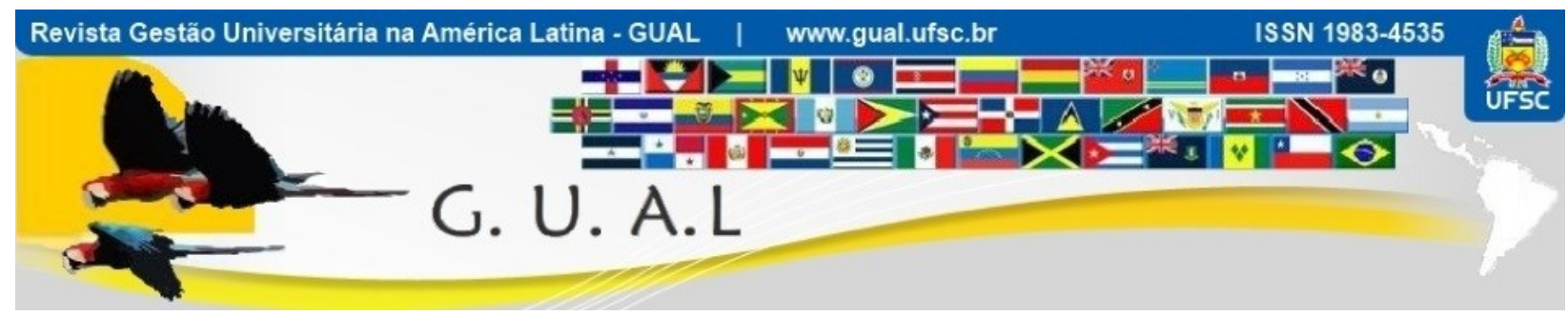

DOI: http://dx.doi.org/10.5007/1983-4535.2015v8n3p219

\title{
ESCOLHA DE CURSOS DE PÓS-GRADUAÇÃO LATO SENSU E OS SEUS FATORES DETERMINANTES
}

CHOICE OF POST GRADUATE COURSES AND ITS DETERMINING FACTORS

\author{
Amire Tauil, Mestre \\ FUCAPE Business School \\ tauil.amire@gmail.com \\ Emerson Wagner Mainardes, Doutor \\ FUCAPE Business School \\ emerson@fucape.br
}

Recebido em 03/dezembro/2014

Aprovado em 19/maio/2015

Sistema de Avaliação: Double Blind Review

Esta obra está sob uma Licença Creative Commons Atribuição-Uso. 


\title{
RESUMO
}

A profissionalização do ramo educacional faz com que as IES (Instituições de Ensino Superior) busquem informações sobre atratividade de seus cursos, ou seja, quais fatores são determinantes para a escolha desta Instituição. Assim, o presente estudo tem como objetivo identificar quais os fatores determinantes na escolha dos alunos por cursos de pós-graduação lato sensu de uma instituição de ensino superior. Para atender ao objetivo foi realizada uma pesquisa descritiva com abordagem quantitativa, em uma instituição de ensino superior de Vitória- Espírito Santo, por meio da aplicação de um questionário a 210 estudantes no seu primeiro dia de aula na pós-graduação. Os dados foram analisados com uso de estatística descritiva e multivariada. Os principais resultados, que contribuem positivamente para a atratividade da IES, estão relacionados a fatores como: estrutura da instituição de ensino superior, por exemplo, segurança do campus e o local de refeição; atualização do curso, professores com vivência no mercado do trabalho e atualização das disciplinas do curso; e motivos pessoais, como a localização da instituição. Estes fatores pode ser um importante indicador para as IES que oferecem curso de pós-graduação.

Palavras-Chave: Marketing educacional. Instituições de Ensino Superior. Pós-graduação Lato Sensu. Atratividade.

\begin{abstract}
The professionalization of the educational branch makes HEIs (Higher Education Institutions) seek information on attractiveness of their courses, that is, which factors are decisive for the choice of this institution. Thus, this study aims to identify the determining factors in the choice of students for post-graduation courses in a higher education institution. To meet the objective of a descriptive research with a quantitative approach was carried out in a higher education institution of Vitória,Espirito Santo, through the application of a questionnaire to 210 students in their first day of class in the graduate school. Data were analyzed using descriptive and multivariate statistics. The main results, which contribute positively to the attractiveness of the HEIs, are related to factors such as: the structure of the higher education institution, for example, campus security and the place of meal; update of the course, teachers with experience in the labor market and updating course subjects; and personal reasons, such as the location of the institution. These factors can be an important indicator for HEIs offering postgraduate course.
\end{abstract}

Keywords: Educational marketing. Graduate Lato Sensu. Attractiveness. 


\section{INTRODUÇÃO}

O modelo de ensino por meio de cursos de pós-graduação lato sensu é relativamente novo no Brasil quando comparado com outros países e, por isso, o tema foi pouco abordado na literatura, apesar de sua relevância. Diversos autores ressaltam a importância da continuidade dos estudos (LEITE; LIMA,1997; MAINARDES, 2007), e a necessidade de investimento contínuo em qualificação do aprendizado (MELO; BORGES, 2007, MANTOVANI, 1995).

O estudo de Mainardes, Deschamps e Domingues, 2006, p. 250, afirma que "a elevação do número de diplomados em graduação criou uma situação em que a certificação deixou de ser um elemento de excelência”. Onde as organizações no Brasil estão exigindo qualificações específicas, o que requer maior aplicabilidade do conhecimento em situações no dia-a-dia (MELO; BORGES, 2007; MEYER, 2003; MANTOVANI, 1995).

A pressão pela atualização e a busca para solução de diversos problemas organizacionais faz com que os profissionais do mercado sejam cobrados continuamente com o intuito de melhorar seu desempenho, visto que fórmulas e soluções do passado já não resolvem os problemas cotidianos atuais (MAINARDES; DOMINGUES, 2011).

Assim, a pós-graduação lato sensu foi criada para os profissionais de nível superior, que tem por objetivo a especialização profissional visando conhecimentos sobre determinada área de forma específica. "São cursos destinados ao aperfeiçoamento e à especialização, nas partes que se compõem um ramo profissional ou científico" (MONTEIRO, 2008, p. 5).

A literatura (KOTLER; FOX, 1995; COBRA; BRAGA, 2004; MENEGUELLI, 2011) indica que a busca contínua do saber atrai estudantes recém-formados e profissionais que desejam o título de especialista dentro de um cenário competitivo de mercado, e essa crescente demanda por especialização levou a muitas IES oferecerem esta modalidade de ensino no Brasil.

O aumento significativo de instituições que oferecem esse tipo de serviço no Brasil, e a facilidade de acesso fazem com que estudos a respeito do marketing educacional venham a se tornar uma ferramenta necessária à gestão da IES, a fim de otimizar a captação de alunos (MENEGHELLI, 2011; COBRA; BRAGA, 2004).

Neste sentido, estudar os fatores determinantes da escolha de uma pós-graduação lato sensu é uma forma de antecipar o conhecimento da gestão sobre as estratégias de atração desses estudantes. Portanto, o presente estudo busca responder à seguinte pergunta: Quais são 
os fatores que determinam a escolha de um aluno por um curso de pós-graduação lato sensu de uma instituição de ensino superior? Desta forma, o objetivo de estudo foi identificar os fatores determinantes na escolha dos alunos por cursos de pós-graduação lato sensu de uma instituição de ensino superior.

Este estudo foi realizado buscando contribuir com a literatura em marketing educacional, visto que a literatura em gestão do marketing das IES, no que tange ao lato sensu, não vem acompanhando a sua evolução de mercado e novos conhecimentos são necessários para apoiar as atividades de marketing de uma IES, sendo esta a principal contribuição teórica pretendida neste estudo. Além disso, ressalta-se a importância do presente estudo para a gestão da IES privada, devido à crescente competitividade no setor.

Para alcançar o objetivo deste estudo, além da introdução, no tópico dois é apresentado o referencial teórico que deu suporte à pesquisa realizada. Em seguida, descrevese a metodologia utilizada. Após esta etapa, são apresentadas as análises realizadas. Finalizase o estudo com as conclusões.

\section{REFERENCIAL TEÓRICO}

\subsection{MARKETING EDUCACIONAL E ATRATIVIDADE DE ALUNOS}

A partir dos anos 90, com a promulgação da Lei de Diretrizes e Bases da Educação (LDB), as características de oferta de cursos no ensino superior foram modificadas. "Após a promulgação da Lei de Diretrizes e Bases da Educação - Lei 9.394/96 o ensino superior expandiu-se de forma acelerada, alterando profundamente o modelo educacional do Brasil” (HOLANDA JUNIOR; FARIAS; GOMES, 2006, p. 103).

Consequentemente, a literatura mais recente já cita o marketing educacional como um elemento fundamental na gestão das IES. O marketing educacional abrange novos campos científicos para continuar a identificar os desejos e necessidades dos compradores e suas exigências, desenvolvendo e desenhando produtos cada vez melhores e tornando seus serviços cada vez mais adequados ao mercado educacional, que se encontra em constante mudança. (ALVES; MAINARDES; RAPOSO, 2010). A utilidade desta área do marketing deve-se às IES necessitarem receber certos recursos através da troca para poderem oferecer os seus serviços. A instituição oferece cursos aos seus mercados e, em troca, recebe os recursos de que necessita. "É esta noção de troca que é central no marketing." (ALVES; MAINARDES; RAPOSO 2010, p.38). 


\section{ESCOLHA DE CURSOS DE PÓS-GRADUAÇÃO LATO SENSU E OS SEUS FATORES \\ DETERMINANTES \\ DOI: http://dx.doi.org/10.5007/1983-4535.2015v8n3p219}

É válido identificar a principal diferença do marketing educacional em relação ao marketing tradicional. O marketing tradicional têm como foco negócios como a indústria e comércio: a definição do produto no processo de troca. No mercado tradicional o produto a ser transacionado é facilmente definido. No marketing educacional, esse "produto" possui variações quanto à visão dos agentes. A visão da Universidade aponta para os cursos oferecidos. Para os estudantes, no entanto, o produto comprado será a qualificação, as perspectivas de carreira, aumento do nível de empregabilidade, entre outros. (ALVES; MAINARDES; RAPOSO, 2010).

Para resumir o presente tópico, de acordo com os estudos realizados (LITTEN, 1980; KOTLER; FOX, 1995; SIRVANCI, 1996; CARVALHO; BERBEL, 2001; ALVES; MAINARDES; RAPOSO, 2010), no quadro 1 são apresentadas as principais diferenças entre o marketing tradicional e o marketing educacional.

\begin{tabular}{l|l|l}
\cline { 2 - 3 } Produto & \multicolumn{1}{c|}{ Marketing Tradicional } & \multicolumn{1}{c}{ Marketing Educacional } \\
\hline Cliente & $\begin{array}{l}\text { Normalmente não há restrição na } \\
\text { aceitação do cliente; }\end{array}$ & $\begin{array}{l}\text { Varia de acordo com os agentes (IES e } \\
\text { alunos). }\end{array}$ \\
\hline Recursos & $\begin{array}{l}\text { Normalmente usam recursos próprios } \\
\text { para a compra; na aceitação do cliente }\end{array}$ & $\begin{array}{l}\text { Podem receber subsídios do governo, } \\
\text { como bolsas de estudos e também podem } \\
\text { receber subsídios dos familiares. }\end{array}$ \\
\hline $\begin{array}{l}\text { Pagamento x } \\
\text { Recebimento do }\end{array}$ & $\begin{array}{l}\text { Normalmente o cliente recebe o } \\
\text { produto que comprou e pagou; }\end{array}$ & $\begin{array}{l}\text { Mesmo que tenha feito o pagamento } \\
\text { integral do curso o aluno pode não } \\
\text { receber o seu diploma ou certificado, } \\
\text { depende dos requisitos mínimos das } \\
\text { disciplinas. }\end{array}$ \\
\hline Processo de Troca & $\begin{array}{l}\text { Pagamento integral do produto; } \\
\text { Fagamento parcial do produto, no ato da } \\
\text { matrícula e financiamento do restante em } \\
\text { mensalidades. }\end{array}$ \\
\hline Fidelidade & $\begin{array}{l}\text { O cliente pode voltar no } \\
\text { estabelecimento comercial por } \\
\text { fidelidade à marca; }\end{array}$ & $\begin{array}{l}\text { O aluno tende a fazer apenas uma } \\
\text { escolha, o custo de mudança é alto e ele } \\
\text { pretende finalizar todos seus estudos } \\
\text { naquela IES. }\end{array}$ \\
\hline $\begin{array}{l}\text { Nem sempre é difícil e não acarreta } \\
\text { consequências no médio e longo } \\
\text { prazo; }\end{array}$ & $\begin{array}{l}\text { Processo difícil, que acarretará sempre em } \\
\text { consequências no médio e longo prazo. }\end{array}$ \\
\hline
\end{tabular}

Quadro 1 Diferenças entre o marketing tradicional e o marketing educacional Fonte: Elaboração Própria

Entre os estudos realizados pelo marketing educacional, destacam-se os estudos de atratividade de alunos (por exemplo, ALFINITO; GRANEMANN, 2003; HOLANDA JUNIOR; GOMES; FARIAS, 2006; SCARPIN; SCHARF; FERNANDES, 2011; AKAREEM; HOSSAIN, 2012). Estas investigações buscam encontrar os atributos e fatores 


\section{ESCOLHA DE CURSOS DE PÓS-GRADUAÇÃO LATO SENSU E OS SEUS FATORES DETERMINANTES \\ DOI: http://dx.doi.org/10.5007/1983-4535.2015v8n3p219}

que levam um aluno a optar por um ou outro curso e/ou IES. Estes estudos têm sido realizados em ambientes de alta competitividade, onde há mais oferta de cursos do que alunos para preencher as vagas, isso vem acontecendo no Brasil desde a promulgação da LDB de 1996. Neste sentido, a partir dos anos 2000, começaram a surgir no Brasil trabalhos acadêmicos que tratam do marketing educacional no que tange aos atributos de escolha por um curso e/ou IES, conforme pode ser observado no quadro 2.

\begin{tabular}{|c|c|c|}
\hline & INDICADORES & AUTORES \\
\hline 1 & Foco no aprendizado; & $\begin{array}{l}\text { THIES ET. AL. (2005); MAINARDES, ALVES \& DOMINGUES } \\
\text { (2011); SCARPIN, DOMINGUES \& SCARPIN (2010) }\end{array}$ \\
\hline 2 & $\begin{array}{l}\text { O curso será difícil e exigirá } \\
\text { esforço por parte do aluno; }\end{array}$ & $\begin{array}{l}\text { THIES ET. AL. (2005); MAINARDES, ALVES \& DOMINGUES } \\
\text { (2011); SCARPIN, DOMINGUES \& SCARPIN (2010) }\end{array}$ \\
\hline 3 & $\begin{array}{l}\text { Alto grau de comprometimento } \\
\text { do aluno; }\end{array}$ & $\begin{array}{l}\text { THIES ET. AL. (2005); MAINARDES, ALVES \& DOMINGUES } \\
\text { (2011); SCARPIN, DOMINGUES \& SCARPIN (2010) }\end{array}$ \\
\hline 4 & $\begin{array}{l}\text { Curso com alto grau de } \\
\text { exigibilidade e cobrança; }\end{array}$ & $\begin{array}{l}\text { THIES ET. AL. (2005); MAINARDES, ALVES \& DOMINGUES } \\
\text { (2011); SCARPIN, DOMINGUES \& SCARPIN (2010) }\end{array}$ \\
\hline 5 & $\begin{array}{l}\text { Utilidade do aprendizado no } \\
\text { trabalho; }\end{array}$ & PIÑOL (2004) ; AKAREEM; HOSSAIN (2012) \\
\hline 6 & As disciplinas serão atualizadas; & PIÑOL (2004) \\
\hline 7 & $\begin{array}{l}\text { Disciplinas serão ligadas à } \\
\text { realidade profissional; }\end{array}$ & PIÑOL (2004) \\
\hline 8 & Grade curricular do curso; & PIÑOL (2004) \\
\hline 9 & Atingir melhores salários; & $\begin{array}{l}\text { ALFINITO \& GRANEMANN (2003); SCARPIN, DOMINGUES \& } \\
\text { SCARPIN (2010) }\end{array}$ \\
\hline 10 & $\begin{array}{l}\text { Valorização no mercado de } \\
\text { trabalho; }\end{array}$ & $\begin{array}{l}\text { HOLANDA JUNIOR,GOMES \& FARIAS (2006); SCARPIN; } \\
\text { SCHARF; FERNANDES (2011); MAINARDES, ALVES \& } \\
\text { DOMINGUES (2011); SCARPIN, DOMINGUES \& SCARPIN } \\
\text { (2010) }\end{array}$ \\
\hline 11 & $\begin{array}{l}\text { Melhores oportunidades de } \\
\text { carreira; }\end{array}$ & $\begin{array}{l}\text { DOMINGUES, SCARPIN \& SCARPIN (2011); AKAREEM; } \\
\text { HOSSAIN (2012) }\end{array}$ \\
\hline 12 & $\begin{array}{l}\text { Melhores oportunidades de } \\
\text { trabalho; }\end{array}$ & $\begin{array}{l}\text { ALFINITO \& GRANEMANN (2003); HOLANDA JUNIOR,GOMES } \\
\text { \& FARIAS (2006); SCARPIN; SCHARF; FERNANDES (2011); } \\
\text { MAINARDES, ALVES \& DOMINGUES (2011); AKAREEM; } \\
\text { HOSSAIN (2012) }\end{array}$ \\
\hline 13 & Cantina; & $\begin{array}{l}\text { ALFINITO \& GRANEMANN (2003); HOLANDA JUNIOR, } \\
\text { GOMES \& FARIAS (2006); BERGAMO ET. AL. (2010); } \\
\text { AKAREEM; HOSSAIN (2012) }\end{array}$ \\
\hline 14 & $\begin{array}{l}\text { Segurança no campus e no } \\
\text { entorno; }\end{array}$ & $\begin{array}{l}\text { MUND, DIUREX \& TONTINI (2001); ALFINITO \& } \\
\text { GRANEMANN (2003); SCARPIN, DOMINGUES \& SCARPIN } \\
\text { (2010); AKAREEM; HOSSAIN (2012) }\end{array}$ \\
\hline 15 & $\begin{array}{l}\text { Equipamentos de audiovisual e } \\
\text { internet nas salas de aula; }\end{array}$ & $\begin{array}{l}\text { ALFINITO \& GRANEMANN (2003); SCARPIN, DOMINGUES \& } \\
\text { SCARPIN (2010) }\end{array}$ \\
\hline 16 & Biblioteca; & $\begin{array}{l}\text { MUND, DIUREX \& TONTINI (2001); ALFINITO \& } \\
\text { GRANEMANN (2003); HOLANDA JUNIOR, GOMES \& FARIAS } \\
\text { (2006); BERGAMO ET. AL. (2010); AKAREEM; HOSSAIN (2012) }\end{array}$ \\
\hline 17 & Laboratórios de informática; & $\begin{array}{l}\text { HOLANDA JUNIOR, GOMES \& FARIAS (2006); BERGAMO ET. } \\
\text { AL. (2010); MAINARDES, ALVES \& DOMINGUES (2011); } \\
\text { SCARPIN, DOMINGUES \& SCARPIN (2010) }\end{array}$ \\
\hline 18 & Laboratórios de prática; & $\begin{array}{l}\text { BERGAMO ET. AL. (2010); MAINARDES, ALVES \& } \\
\text { DOMINGUES (2011 SCARPIN, DOMINGUES \& SCARPIN (2010) }\end{array}$ \\
\hline
\end{tabular}




\section{ESCOLHA DE CURSOS DE PÓS-GRADUAÇÃO LATO SENSU E OS SEUS FATORES DETERMINANTES \\ DOI: http://dx.doi.org/10.5007/1983-4535.2015v8n3p219}

\begin{tabular}{|c|c|c|}
\hline 19 & $\begin{array}{l}\text { Infraestrutura adequada para as } \\
\text { necessidades dos alunos; }\end{array}$ & $\begin{array}{l}\text { MUND, DIUREX \& TONTINI (2001); ALFINITO \& } \\
\text { GRANEMANN (2003); HOLANDA JUNIOR, GOMES \& FARIAS } \\
\text { (2006); MAINARDES, ALVES \& DOMINGUES (2011); } \\
\text { AKAREEM; HOSSAIN (2012) }\end{array}$ \\
\hline 20 & $\begin{array}{l}\text { Localizado próximo a residência } \\
\text { do aluno; }\end{array}$ & $\begin{array}{l}\text { ALFINITO \& GRANEMANN, (2003); ALFINITO \& } \\
\text { GRANEMANN, (2003); }\end{array}$ \\
\hline 21 & $\begin{array}{l}\text { Localizado próximo do local de } \\
\text { trabalho do aluno; }\end{array}$ & $\begin{array}{l}\text { MUND, DIUREX \& TONTINI, (2001); HOLANDA JUNIOR, } \\
\text { GOMES \& FARIAS (2006); BERGAMO ET. AL. (2010); }\end{array}$ \\
\hline 22 & Local seguro; & $\begin{array}{l}\text { MUND, DIUREX \& TONTINI, (2001); HOLANDA JUNIOR, } \\
\text { GOMES \& FARIAS (2006); BERGAMO ET. AL. (2010); }\end{array}$ \\
\hline 23 & Acesso ao local de aula; & $\begin{array}{l}\text { ALFINITO \& GRANEMANN, (2003); ALFINITO \& } \\
\text { GRANEMANN, (2003); }\end{array}$ \\
\hline 24 & $\begin{array}{l}\text { Valor das mensalidades } \\
\text { adequado ao curso escolhido; }\end{array}$ & $\begin{array}{l}\text { TREVISAN (2001) ALFINITO \& GRANEMANN, (2003); MUND, } \\
\text { DIUREX \& TONTINI, 2001; BERGAMO ET. AL. (2010) }\end{array}$ \\
\hline 25 & Mensalidades atraentes; & $\begin{array}{l}\text { TREVISAN (2001) ALFINITO \& GRANEMANN, (2003); MUND, } \\
\text { DIUREX \& TONTINI, 2001; BERGAMO ET. AL. (2010) }\end{array}$ \\
\hline 26 & Mensalidades acessíveis; & $\begin{array}{l}\text { TREVISAN (2001) ALFINITO \& GRANEMANN, (2003); MUND, } \\
\text { DIUREX \& TONTINI, 2001; BERGAMO ET. AL. (2010) }\end{array}$ \\
\hline 27 & Duração do curso; & $\begin{array}{l}\text { MAINARDES, DESCHAMPS \& DOMINGUES (2006); ALFINITO } \\
\text { \& GRANEMANN, (2003) }\end{array}$ \\
\hline 28 & Periodicidade de aulas; & $\begin{array}{l}\text { MAINARDES, DESCHAMPS \& DOMINGUES (2006); ALFINITO } \\
\text { \& GRANEMANN, (2003) }\end{array}$ \\
\hline 29 & $\begin{array}{l}\text { Horário do curso adequado ao } \\
\text { aluno; }\end{array}$ & $\begin{array}{l}\text { MAINARDES, DESCHAMPS \& DOMINGUES (2006); ALFINITO } \\
\text { \& GRANEMANN, (2003) }\end{array}$ \\
\hline 30 & $\begin{array}{l}\text { O curso adaptado (horários, } \\
\text { valores, dias) ao aluno; }\end{array}$ & $\begin{array}{l}\text { MAINARDES, DESCHAMPS \& DOMINGUES (2006); ALFINITO } \\
\text { \& GRANEMANN, (2003) }\end{array}$ \\
\hline 31 & Faculdade reconhecida; & $\begin{array}{l}\text { SCARPIN; SCHARF \& FERNANDES, (2011); BERGAMO ET. AL. } \\
\text { (2010); PAMPALONI (2010); MAINARDES, ALVES \& } \\
\text { DOMINGUES (2011); SCARPIN, DOMINGUES \& SCARPIN } \\
(2010)\end{array}$ \\
\hline 32 & $\begin{array}{l}\text { Instituição é bem vista pelos } \\
\text { amigos e familiares. }\end{array}$ & $\begin{array}{l}\text { SCARPIN; SCHARF \& FERNANDES, (2011); BERGAMO ET. AL. } \\
\text { (2010); PAMPALONI (2010); MAINARDES, ALVES \& } \\
\text { DOMINGUES (2011); SCARPIN, DOMINGUES \& SCARPIN } \\
(2010)\end{array}$ \\
\hline 33 & Marca da IES; & $\begin{array}{l}\text { ALFINITO \& GRANEMANN (2003); SILVA, (2005); BENNETT \& } \\
\text { ALI-CHOUDHURY (2009); FERNANDES, SCARPIN \& SCHARF } \\
\text { (2011); BERGAMO ET. AL. (2010); }\end{array}$ \\
\hline 34 & $\begin{array}{l}\text { Imagem positiva perante a } \\
\text { sociedade; }\end{array}$ & $\begin{array}{l}\text { ALFINITO \& GRANEMANN, 2003; SILVA, (2005); BENNETT \& } \\
\text { ALI-CHOUDHURY (2009); PAMPALONI (2010); FERNANDES, } \\
\text { SCARPIN \& SCHARF (2011); BERGAMO ET. AL. (2010); } \\
\text { SCARPIN, DOMINGUES \& SCARPIN (2010) }\end{array}$ \\
\hline 35 & $\begin{array}{l}\text { Professores com vivência no } \\
\text { mercado de trabalho. }\end{array}$ & THIES ET. AL. (2005); PIÑOL (2004) \\
\hline 36 & Professores dinâmicos; & THIES ET. AL. (2005); PIÑOL (2004) \\
\hline 37 & Aulas motivantes; & THIES ET. AL. (2005); PIÑOL (2004) \\
\hline 38 & $\begin{array}{l}\text { Relações entre a teoria e a } \\
\text { prática; }\end{array}$ & THIES ET. AL. (2005); PIÑOL (2004) \\
\hline 39 & Professores disponíveis; & THIES ET. AL. (2005); PIÑOL (2004) \\
\hline 40 & Professores terão alta titulação; & THIES ET. AL. (2005); PIÑOL (2004) \\
\hline 41 & Material de estudo; & THIES ET. AL. (2005); PIÑOL (2004) \\
\hline 42 & Corpo docente qualificado. & THIES ET. AL. (2005); PIÑOL (2004) \\
\hline
\end{tabular}

Quadro 2 Principais atributos de atração de estudantes

Fonte: Elaboração própria. 


\section{ESCOLHA DE CURSOS DE PÓS-GRADUAÇÃO LATO SENSU E OS SEUS FATORES

\subsection{A PÓS-GRADUAÇÃO LATO SENSU}

Os critérios para o funcionamento da pós-graduação no Brasil foram estabelecidos através do Parecer 977/65 do CFE. Nesse momento, “os cursos de pós-graduação foram estruturados em níveis e finalidades diferenciados: stricto e lato sensu." (SCARPIN; DOMINGUES; SCARPIN, 2010, p. 5). Entende-se como pós-graduação strictu sensu, os cursos de mestrado e doutorado, e a pós-graduação lato sensu pode ser definida como o curso que se segue à graduação, seja ele de qualquer natureza, sendo representada pelos cursos de especialização (MONTEIRO, 2008).

"As pós-graduações lato sensu compreendem programas de especialização e incluem os cursos designados como MBA - Master Business Administration. Possuem duração mínima de 360 horas e ao final do curso o aluno obterá certificado, não diploma." (MEC, 2007). A pós-graduação lato sensu objetiva a especialização profissional, pois "sua meta é o domínio científico e técnico de certa e limitada área do saber ou profíssão e como são cursos voltados predominantemente ao mercado de trabalho, os mesmos não conferem grau acadêmico" (SCARPIN; DOMINGUES; SCARPIN, 2010; p. 5). Porém, esses cursos oferecem oportunidade de educação especializada. (MONTEIRO, 2008).

\section{METODOLOGIA}

Considerando que o principal objetivo deste estudo foi identificar os fatores determinantes na escolha dos alunos por cursos de pós-graduação lato sensu de uma instituição de ensino superior, escolheu-se realizar uma pesquisa quantitativa, descritiva e transversal, por meio de levantamento de dados primários, com a aplicação de questionário com perguntas predominantemente fechadas (HAIR JR. et al., 2005). Para a realização da investigação, foi escolhida uma IES privada do Espírito Santo.

A escolha da IES pesquisada se deu pelo fato da mesma estar hoje entre as maiores IES privadas do Brasil, contando com mais de setenta unidades em todo país (INEP, 2008). A unidade do Espírito Santo permitiu a realização da pesquisa em suas dependências, sendo que a mesma oferece sete cursos presenciais de pós-graduação lato sensu, contando com 275 alunos recém matriculados, população alvo deste estudo. Os cursos oferecidos pela unidade do Espírito Santo são: Direito e Processo do Trabalho e Direito Previdenciário, Direito Público: administrativo, constitucional e tributário, Fisioterapia Dermato Funcional, Gestão Estratégica de Marketing e Vendas, Logística Empresarial, MBA em Controladoria 


\section{ESCOLHA DE CURSOS DE PÓS-GRADUAÇÃO LATO SENSU E OS SEUS FATORES \\ DOI: http://dx.doi.org/10.5007/1983-4535.2015v8n3p219}

Estratégica e Finanças, MBA em Gestão de Pessoas e Treinamento e Biodinâmica aplicado à qualidade de vida. Essa característica justificou a escolha por essa IES, pois nota-se que são raras as pesquisas sobre lato sensu no Brasil que pesquisaram cursos de três grandes áreas do conhecimento (gestão, saúde e direito). Considerando a população em questão, foram coletados dados junto a 210 alunos ingressantes em cursos de pós-graduação lato sensu, pois os mesmos estavam presentes em sala de aula no primeiro dia do curso. A escolha desta população deve-se ao fato de terem recém contratado o serviço de pós-graduação lato sensu e ainda terem em mente os motivos de sua escolha.

Desta forma, foram realizadas as coletas de dados em duas etapas. A primeira etapa, no intuito de construir o questionário, consistiu da aplicação de um questionário inicial a seis alunos de cada turma de pós-graduação lato sensu, porém esses alunos já estavam matriculados desde o início do ano de 2012 na IES, não fazendo parte da população alvo deste estudo. Pretendeu-se identificar possíveis dúvidas dos respondentes sobre o conteúdo das perguntas e realizar uma análise fatorial exploratória.

Os 42 atributos foram transformados em afirmações acompanhadas da escala de Likert de cinco pontos. As respostas variaram entre "discordo totalmente" até "concordo totalmente". A finalidade desta etapa foi agrupar os 42 indicadores (quadro 2) em grupos comuns, favorecendo as análises dos critérios dos alunos quanto à escolha de um curso de pós-graduação lato sensu. Ao invés de analisar 42 atributos separadamente, pode-se analisar grupos de atributos comuns, que, por serem em menor número, favorecem os gestores das IES nas suas atividades de atração de alunos.

No total, foram escolhidos aleatoriamente 60 alunos para responder esse primeiro questionário com 42 questões (de acordo com o quadro 2 do referencial teórico), que foram ordenadas em ordem alfabética. Esse questionário, com 42 questões sobre atratividade encontradas na literatura, foi aplicado no dia 11 de setembro de 2012. A análise fatorial exploratória permitiu o agrupamento dos 42 atributos da presente pesquisa em oito fatores: $\mathrm{O}$ primeiro fator ficou com oito atributos - Características do curso (Y1); O segundo fator ficou com sete atributos - Aprendizado (Y2); O terceiro fator ficou com sete atributos - Imagem da IES (Y3); O quarto fator ficou com cinco atributos - Empregabilidade (Y4); O quinto fator ficou com cinco atributos - Estrutura da organização (Y5); O sexto fator ficou com quatro atributos - Estrutura do curso (Y6); O sétimo fator ficou com quatro atributos - Fatores pessoais (Y7) e; O oitavo e último fator ficou com dois atributos - Atualização do curso (Y8). 
Após a análise fatorial, o conteúdo do questionário foi reformulado de acordo com os oito fatores e foi incluída uma questão geral sobre atratividade dos cursos analisados (variável Y). Também foi incluída, ao final do questionário a caracterização do respondente: curso de pós-graduação escolhido, idade, gênero, estado civil, filhos, função profissional e setor de atuação. A caracterização dos respondentes pode auxiliar no agrupamento de percepções de alunos com características comuns (por exemplo, por curso, por idade, área do conhecimento, entre outros).

Assim, o questionário foi considerado pronto para ser aplicado para população alvo. Dos 275 alunos recém matriculados que compuseram a população alvo da pesquisa, todos que compareceram ao primeiro dia letivo da pós-graduação, em 13 de outubro de 2012, ou seja, 210 alunos, foram levados ao laboratório de informática do campus para responder o questionário final.

Para analisar os dados, utilizaram-se proporções de respostas (para caracterizar os respondentes), estatísticas descritivas (média e desvio padrão) e regressão linear múltipla. Nesta última, a variável dependente foi à atratividade do curso (Y) e as variáveis independentes foram: características gerais do curso (Y1), aprendizado (Y2), imagem (Y3), empregabilidade (Y4), estrutura da instituição (Y5), estrutura de apoio ao curso (Y6), motivos pessoais (Y7) e atualização do curso (Y8).

\section{ANÁLISE DOS DADOS}

A análise dos dados foi dividida em três momentos: caracterização da amostra, estatísticas descritivas e, por fim, a regressão linear múltipla. Inicialmente caracterizou a amostra, como pode ser observado na tabela 1 .

Tabela 1 Caracterização da amostra dos alunos

\begin{tabular}{c|l|c|c}
\hline \multirow{2}{*}{ Gênero } & Masculino & $\mathrm{N}$ & $\%$ \\
& Feminino & 79 & $37,62 \%$ \\
& 20 a 26 anos & 131 & $62,38 \%$ \\
\hline \multirow{2}{*}{ Faixa de Idade } & 27 a 33 anos & 69 & $32,86 \%$ \\
& 34 a 40 anos & 83 & $39,52 \%$ \\
& 41 a 47 anos & 31 & $14,76 \%$ \\
& 48 a 54 anos & 14 & $6,67 \%$ \\
& 55 a 68 anos & 11 & $5,24 \%$ \\
\hline \multirow{2}{*}{ Estado Civil } & Solteiro & 2 & $0,96 \%$ \\
\hline & Casado & 81 & $51,90 \%$ \\
& & & $38,57 \%$ \\
\hline
\end{tabular}




\begin{tabular}{c|l|c|c}
\hline & Separado & 11 & $5,24 \%$ \\
& União Estável & 9 & $4,29 \%$ \\
\hline \multirow{4}{*}{$N^{o}$ de Filhos } & Nenhum & 154 & $73,33 \%$ \\
& Um & 30 & $14,29 \%$ \\
& Dois & 21 & $10,00 \%$ \\
& Três & 4 & $1,90 \%$ \\
& Mais de três & 1 & $0,48 \%$ \\
\hline \multirow{5}{*}{ Local de Trabalho } & Administração Pública & 21 & $10,00 \%$ \\
& Iniciativa Privada (indústria) & 31 & $14,76 \%$ \\
& Iniciativa Privada (Comércio) & 36 & $17,14 \%$ \\
& Iniciativa Privada (Serviços) & 94 & $44,76 \%$ \\
& Terceiro Setor & 6 & $2,86 \%$ \\
& Não trabalho & 10 & $4,76 \%$ \\
& Outros & 12 & $5,71 \%$ \\
\hline \multirow{5}{*}{ Função Profissional } & Operacional (sem subordinados) & 75 & $35,71 \%$ \\
& Supervisão & 40 & $19,05 \%$ \\
& Gerência & 28 & $13,33 \%$ \\
& Alta Gerência & 4 & $1,90 \%$ \\
& Direção & 2 & $0,95 \%$ \\
& Proprietário/Acionista & 13 & $6,19 \%$ \\
& Não trabalho & 9 & $4,29 \%$ \\
& Outros & 39 & $18,57 \%$ \\
\hline Total & & 210 & $100,00 \%$ \\
\hline
\end{tabular}

$\mathrm{n}$ - amostra; \% - freqüência relativa.

Fonte: elaboração própria

A Tabela 2 permite distinguir o perfil dos respondentes quanto aos dados sócio demográficos, a qual predominaram as seguintes características: sexo feminino com $62,38 \%$; em relação faixa de idade, $32,86 \%$ dos respondentes têm entre 20 a 26 anos e 39,52\% têm entre 27 e 33 anos; quanto ao estado civil, 51,90\% são solteiros; e em relação ao número de filhos, 73,33\% não têm filhos. Foi possível identificar, ainda, que 44,76\% da amostra trabalha na iniciativa privada, no setor de serviço; no que diz respeito à função profissional, quase 55\% dos respondentes têm cargo operacional (sem subordinados) ou de supervisão.

Esse resultado da função profissional dos alunos que optaram por fazer cursos de pósgraduação lato sensu, corrobora com as análises feitas pelos autores Mainardes, Deschamps e Domingues (2006, p. 12), que afirmam que os egressos "buscam, em cursos de pós-graduação lato sensu, os conhecimentos que lhes faltam para estarem melhor preparados para o mercado de trabalho". Nota-se que quase 55\% dos respondentes trabalham no setor operacional ou de supervisão, ou seja, ainda não atingiram níveis profissionais mais altos como alta gerência, 


\section{ESCOLHA DE CURSOS DE PÓS-GRADUAÇÃO LATO SENSU E OS SEUS FATORES \\ DETERMINANTES \\ DOI: http://dx.doi.org/10.5007/1983-4535.2015v8n3p219}

portanto, pode-se afirmar que essa busca contínua do saber atrai alunos que almejam ascensão profissional.

A análise seguinte foram às estatísticas descritivas. Os resultados obtidos podem ser observados na tabela 2 .

Tabela 2 Estatística descritiva dos fatores

\begin{tabular}{|c|c|c|}
\hline Indicadores "CARACTERÍSTICAS DO CURSO" & Média & Desvio Padrão \\
\hline X1A - Professores disponíveis & 3,80 & 1,02 \\
\hline X1B - Relações entre a teoria e a prática & 4,40 & 0,82 \\
\hline $\mathrm{X} 1 \mathrm{C}$ - Utilidade do aprendizado no trabalho & 4,58 & 0,73 \\
\hline X1D - Professores dinâmicos & 4,14 & 0,75 \\
\hline X1E - Periodicidade das aulas & 4,25 & 1,01 \\
\hline X1F - Duração do curso & 4,12 & 0,97 \\
\hline X1G - Disciplinas ligadas à realidade profissional & 4,34 & 0,74 \\
\hline $\mathrm{X} 1 \mathrm{H}$ - Horário do curso adequado ao aluno & 3,92 & 1,10 \\
\hline Y1 - Questão geral características do curso & 4,10 & 1,09 \\
\hline Indicadores “APRENDIZADO” & Média & Desvio Padrão \\
\hline $\mathrm{X} 2 \mathrm{~A}-$ Aulas motivantes & 4,03 & 0,89 \\
\hline X2B - Alto grau de comprometimento do aluno & 4,43 & 0,72 \\
\hline X2C - Curso será difícil e exigirá esforço por farte do aluno & 4,10 & 0,90 \\
\hline X2D - Material de estudo & 4,31 & 0,84 \\
\hline X2E - Foco no aprendizado & 4,27 & 0,85 \\
\hline $\mathrm{X} 2 \mathrm{~F}$ - Professores terão alta titulação & 4,25 & 0,92 \\
\hline $\mathrm{X} 2 \mathrm{G}$ - Curso com alto grau de exibilidade e cobrança & 4,00 & 0,96 \\
\hline Y2 - Questão geral aprendizado & 4,27 & 0,88 \\
\hline Indicadores "IMAGEM" & Média & Desvio Padrão \\
\hline X3A - Corpo docente qualificado & 4,37 & 0,77 \\
\hline X3B - Imagem positiva da IES perante à sociedade & 4,18 & 0,90 \\
\hline X3C - Instituição é bem vista pelos amigos e familiares & 4,10 & 0,89 \\
\hline X3D - Marca da IES & 4,09 & 0,95 \\
\hline X3E - Faculdade é reconhecida no Brasil & 4,40 & 0,80 \\
\hline $\mathrm{X} 3 \mathrm{~F}$ - Valor das mensalidades adequado ao curso escolhido & 4,24 & 0,89 \\
\hline $\mathrm{X} 3 \mathrm{G}$ - A grade curricular do curso & 4,15 & 1,01 \\
\hline Y3 - Questão geral imagem & 3,60 & 1,13 \\
\hline Indicadores "EMPREGABILIDADE" & Média & Desvio Padrão \\
\hline $\mathrm{X} 4 \mathrm{~A}$ - Atingir melhores salários & 4,44 & 0,70 \\
\hline X4B - Melhores oportunidades de carreira & 4,55 & 0,66 \\
\hline X4C - Valorização no mercado de trabalho & 4,36 & 0,87 \\
\hline X4D - Melhores oportunidades de trabalho & 4,54 & 0,66 \\
\hline X4E - Mensalidades acessíveis & 4,18 & 0,93 \\
\hline Y4 - Questão geral empregabilidade & 4,22 & 0,82 \\
\hline Indicadores "ESTRUTURA DA IES" & Média & Desvio Padrão \\
\hline X5A-Biblioteca & 4,20 & 0,84 \\
\hline X5B - Segurança no Campus e no entorno & 4,22 & 0,82 \\
\hline X5C - Cantina & 4,20 & 0,95 \\
\hline X5D - Infraestrutura adequada às necessidades dos alunos & 4,26 & 0,88 \\
\hline X5E - Local seguro & 3,99 & 1,10 \\
\hline Y5 - Questão geral estrutura da IES & 3,53 & 1,11 \\
\hline Indicadores "ESTRUTURA DE APOIO AO CURSO" & Média & Desvio Padrão \\
\hline X6A - Laboratórios de informática & 4,02 & 0,93 \\
\hline X6B - Equipamentos de audiovisual e internet nas salas de aula & 4,23 & 0,89 \\
\hline $\mathrm{X} 6 \mathrm{C}$ - Laboratórios de prática & 3,83 & 1,06 \\
\hline X6E - Curso adaptado (horário, dias) ao aluno & 4,03 & 0,93 \\
\hline
\end{tabular}




\section{ESCOLHA DE CURSOS DE PÓS-GRADUAÇÃO LATO SENSU E OS SEUS FATORES \\ DOI: http://dx.doi.org/10.5007/1983-4535.2015v8n3p219}

\begin{tabular}{lcc} 
Y6 - Questão geral estrutura de apoio & 3,55 & 1,13 \\
\hline Indicadores "MOTIVOS PESSOAIS" & Média & Desvio Padrão \\
\hline X7A - Acesso ao local de aula & 3,84 & 1,33 \\
X7B - Localizado próximo a residência do aluno & 3,27 & 1,65 \\
X7C - Mensalidades atraentes & 3,90 & 1,12 \\
X7D - Localizado próximo ao trabalho do aluno & 2,67 & 1,60 \\
\hline Y7 - Questão geral motivos pessoais & 3,69 & 1,33 \\
\hline Indicadores "ATUALIZAÇÃO DO CURSO" & Média & Desvio Padrão \\
\hline X8A - As disciplinas serão atualizadas & 4,47 & 0,75 \\
X8B - Professores com vivência no mercado de trabalho & 4,54 & 0,71 \\
\hline Y8 - Questão geral atualização do curso & 4,16 & 1,01 \\
\hline
\end{tabular}

Fonte: Elaboração própria

$\mathrm{Na}$ análise descritiva dos fatores e seus indicadores, foi realizada uma análise de médias e desvios padrão. Percebeu-se médias superiores a 4,0, com desvio padrão inferior a 1,0, em todos os indicadores ligados aos fatores Aprendizado, Imagem, Empregabilidade e Atualização do Curso. Isso indica que esses fatores são características que os alunos recémmatriculados em cursos de pós-graduação lato sensu consideram importantes, tendo certa unanimidade de respostas.

Sobre o fator Aprendizado, pode-se afirmar que, conforme analisado anteriormente nos dados da função profissional dos alunos que optaram por fazer cursos de pós-graduação lato sensu, nota-se que quase $55 \%$ dos respondentes trabalham no setor operacional ou de supervisão, ou seja, ainda não atingiram níveis profissionais mais altos como alta gerência. Portanto, pode-se afirmar que o aprender para esses alunos é muito importante e está relacionado à sua vontade de crescer profissionalmente.

Sobre o fator Imagem, para SCARPIN; SCHARF; FERNANDES (2011), a relevância da marca de uma IES para criar valor e diferencial torna-se importante no Brasil, principalmente a partir dos anos 90, pois "tem aumentado a oferta de cursos de especialização, permitindo a opção de escolha pelos estudantes e, também, a maior concorrência entre as IES. Essas por sua vez possuem um produto intangível, baseado no conhecimento". Segundo os mesmos autores, "uma boa marca deve possuir um conjunto de atributos que façam com que os consumidores recomendem-na positivamente a outros" (p. 5), além disso, eles afirmam que "as informações de amigos são fatores importantes para a escolha de uma IES" (SCARPIN; SCHARF; FERNANDES, 2011, p. 9). Os resultados obtidos aqui confirmam o encontrado anteriormente pelos autores citados.

Sobre o fator Empregabilidade, pode-se considerar que estudos anteriores confirmam os resultados aqui encontrados, pois, "diante de um mercado mais restrito, os jovens têm 
buscado na qualificação profissional, principalmente através de cursos de pós-graduação, a independência e participação social". (MELO; BORGES, 2007, p.1). "Se antes com a posse de diploma de curso superior era associada à garantia de vaga no mercado de trabalho e à obtenção de um determinado status social, hoje isso está deixando de ser verdadeiro". (GIOTTO; GOMES; DOMINGUES, 2007, p. 2). Por isso, a pós-graduação lato sensu tem uma forte ascensão, principalmente a partir dos anos 1980 (MONTEIRO, 2008).

Sobre o fator Atualização do curso, esse resultado também pode ser evidenciado nos estudos anteriores realizados por Piñol (2004). Nos três cursos estudados pelo autor, o único atributo que foi unânime nas respostas, como sendo o mais valorizado pelos alunos dos cursos, foi à composição e atualização das disciplinas. (PIÑOL, 2004). Ainda de acordo com o estudo desse autor, para o curso de Gestão Empresarial, além da composição e atualização das disciplinas, o atributo mais valorizado pelos alunos desse curso foi o de professores com vivência no mercado de trabalho (PIÑOL, 2004).

Ao avaliar a Atratividade geral do curso, observou-se média de 4,26 e desvio padrão 0,85. Pode-se perceber, portanto, uma média alta com baixa dispersão de respostas, o que significa certa unanimidade. Esperava-se que a atratividade dos cursos de pós-graduação lato sensuda IES estudada tivesse uma média alta devido ao público alvo da pesquisa ter sido atraído para cursos de pós-graduação dessa IES. Assim, ressalta-se a importância de encontrar os fatores determinantes desta escolha, alvo da análise final.

Desta forma, realizou-se a regressão linear múltipla, tendo a variável geral Atratividade do curso como variável dependente e os oito fatores com variáveis independentes. Os resultados obtidos encontram-se nas tabelas 4 e 5.

Nota-se a partir das tabelas 3 e 4, o poder de explicação do modelo estimado (Adjusted $R$ Square), cujas variáveis Y5 (estrutura da IES), Y7 (atualização do curso) e Y8 (motivos pessoais) explicam 19\% da variabilidade da variável dependente, Atratividade pelo curso (Y). Nos quesitos que validam o modelo, todos os pressupostos foram satisfeitos. 


\section{ESCOLHA DE CURSOS DE PÓS-GRADUAÇÃO LATO SENSU E OS SEUS FATORES \\ DETERMINANTES \\ DOI: http://dx.doi.org/10.5007/1983-4535.2015v8n3p219}

Tabela 3 Regressão múltipla variável dependente atratividade do curso

\begin{tabular}{|c|c|c|c|c|c|c|c|c|c|}
\hline \multirow[b]{2}{*}{$\mathrm{R}$} & \multirow[b]{2}{*}{ R Square } & \multirow[b]{2}{*}{$\begin{array}{l}\text { Adjusted } \\
\text { R Square }\end{array}$} & \multirow{2}{*}{$\begin{array}{l}\text { Std. } \\
\text { Error of } \\
\text { the } \\
\text { Estimate }\end{array}$} & \multicolumn{5}{|c|}{ ChangeStatistics } & \multirow[b]{2}{*}{$\begin{array}{l}\text { Durbin- } \\
\text { Watson }\end{array}$} \\
\hline & & & & $\begin{array}{l}\text { R Square } \\
\text { Change }\end{array}$ & $\begin{array}{l}\mathrm{F} \\
\text { Change }\end{array}$ & df1 & $\mathrm{df} 2$ & $\begin{array}{l}\text { Sig. F } \\
\text { Change }\end{array}$ & \\
\hline 0,45 & 0,20 & 0,19 & 0,77 & 0,02 & 4,53 & 1 & 206 & 0,03 & 1,79 \\
\hline
\end{tabular}

Predictors: (Constant), Y5 - Questão Geral Estrutura, Y8 - Questão Geral Atualização do Curso, Y7 - Questão Geral Motivos Pessoais

DependentVariable: Y - Atratividade pelo Curso

Método de estimação: Stepwise

Testes de validez:

- ANOVA: significativo

- Teste de Aleatoriedade: Aceita a hipótese de aleatoriedade

- Teste de Aderência Kolmogorov-Smirnov: Aceita hipótese de aderência a distribuição normal

- Teste de Homocedasticidade: Aceita a hipótese de homocedasticidade

Fonte: Elaboração própria

Tabela 4 Coeficientes estimados para o modelo geral

\begin{tabular}{|c|c|c|c|c|c|c|c|}
\hline \multirow[t]{2}{*}{ Model } & \multicolumn{2}{|c|}{$\begin{array}{l}\text { Unstandardized } \\
\text { Coefficients }\end{array}$} & \multirow{2}{*}{$\begin{array}{l}\text { Standardized } \\
\text { Coefficients } \\
\text { Beta }\end{array}$} & & & \multicolumn{2}{|c|}{$\begin{array}{l}\text { Collinearity } \\
\text { Statistics }\end{array}$} \\
\hline & $\mathrm{B}$ & Std. Error & & $\mathrm{t}$ & Sig. & TOL & VIF \\
\hline (Constant) & 2,55 & 0,25 & & 9,99 & $0,00^{*}$ & & \\
\hline Y5 - Questão Geral Estrutura & 0,19 & 0,06 & 0,25 & 3,44 & $0,00^{*}$ & 0,75 & 1,34 \\
\hline $\begin{array}{llll}3 & \text { Y8 - } & \text { Questão } & \text { Geral } \\
& \text { atualização do Curso } & \end{array}$ & 0,17 & 0,06 & 0,20 & 2,82 & $0,01^{*}$ & 0,76 & 1,32 \\
\hline $\begin{array}{l}\text { Y7 - Questão Geral Motivos } \\
\text { Pessoais }\end{array}$ & 0,09 & 0,04 & 0,14 & 2,13 & $0,03^{*}$ & 0,93 & 1,08 \\
\hline
\end{tabular}

Dependent Variable: Y - Atratividade pelo Curso

(*) Significativo ao nível de 5\%

Fonte: Elaboração própria

Nota-se a partir das tabelas 3 e 4, o poder de explicação do modelo estimado (Adjusted $R$ Square), cujas variáveis Y5 (estrutura da IES), Y7 (atualização do curso) e Y8 (motivos pessoais) explicam 19\% da variabilidade da variável dependente, Atratividade do curso (Y).

Nos quesitos que validam o modelo, todos os pressupostos foram satisfeitos. Portanto, diante dos resultados observados, constata-se que os fatores que podem explicar a escolha (atração) dos cursos de pós-graduação lato sensuda IES investigada foram: 1) A estrutura da instituição, 2) A atualização do curso e 3) Os motivos pessoais.

\section{Estrutura da IES}

A estrutura física da faculdade foi um fator determinante para a escolha por cursos na IES estudada. Conforme explicado anteriormente na análise descritiva dos fatores, os autores 
Holanda Jr., Farias e Gomes (2006) identificaram que a estrutura física demonstrou ter uma forte influência no índice de evasão dos alunos de IES privadas.

Por isso, é importante que a IES tenha uma estrutura física adequada para receber seus alunos. Ainda corroborando com o resultado encontrado no presente estudo, Alfinito e Granemann (2003) identificaram, dentre os diversos atributos de escolha de uma IES, que o atributo infraestrutura foi o mais importante.

Por exemplo, dentro da variável estrutura da IES, a cantina se mostrou como um indicador relevante para a escolha por essa IES. No caso da pós-graduação lato sensu, pode existir uma maior preocupação por parte dos alunos com suas refeições, devido, principalmente, ao fato do aluno passar todo o dia na IES. No quesito "IES localizada em local seguro", esse resultado também pode ser evidenciado no estudo de Scarpin, Domingues e Scarpin (2010). Os autores identificaram os fatores de atração como diferencial competitivo nos cursos de pós-graduação lato sensu em uma Universidade de Blumenau e, entre os fatores, em sua conclusão, os autores ressaltam o fator Segurança no Campus como diferencial competitivo para a IES. O mesmo se aplica aos demais indicadores deste fator, visto que todos os indicadores receberam notas altas.

\section{Atualização do curso}

Com relação à atualização dos cursos de pós-graduação lato sensu, pode-se afirmar que "a sociedade atual, baseada em alta tecnologia, destaca-se pelo capital intelectual, um fator considerado estratégico nas organizações. Isso coloca as IES no centro das mudanças”. (MEYER, 2003, p. 176). Os alunos que buscam cursos de pós-graduação lato sensu estão em busca de ascensão profissional e precisam especializar-se. Isso ficou claro nas análises descritivas do fator empregabilidade e na análise das funções profissionais dos alunos, onde quase 55\% ainda não atingiram níveis de Gerência ou Alta gerência.

"Elevam-se os índices de escolaridade exigidos pelos diversos segmentos da economia no Brasil” (MEYER, 2003; p. 174), e, por isso, a atualização do curso, com destaque para as disciplinas e seus tópicos atualizados, se mostraram determinantes para a escolha por cursos de pós-graduação lato sensu. 
Motivos Pessoais

Os motivos pessoais foram um dos fatores mais relevantes para a escolha por cursos de pós-graduação lato sensu na IES estudada. Isso pode indicar que a faculdade onde foi feito o presente estudo pode ser classificada como faculdade "conveniente".

De acordo com Cobra e Braga (2004), a tríade acesso, localização e preço denominados no presente estudo como indicadores do fator "motivos pessoais", são mais importantes para as faculdades que não têm um forte diferencial competitivo. Para os autores, esse tipo de instituição é chamada faculdade "conveniente". "A maior parte das IES do Brasil se classificam nessa categoria (convenientes)". A qualidade dos cursos nesses tipos de IES é variável, portanto, os seus cursos não tem um forte diferencial competitivo (COBRA; BRAGA, 2004, p. 41).

\section{CONCLUSÕES}

Realizou-se esta pesquisa com o objetivo de identificar os fatores determinantes na escolha dos alunos por cursos de pós-graduação lato sensu de uma instituição de ensino superior. O estudo mostrou que os atributos "estrutura da IES", "motivos pessoais" e "atualização do curso" foram determinantes para a escolha dos alunos pelos cursos da IES pesquisada, sendo um importante indicador para as IES que oferecem este tipo de curso.

Quanto aos alunos, verificou-se que $62,4 \%$ do público é de gênero feminino, 72,4\% dos alunos recém matriculados têm entre 20 a 33 anos de idade, 51,9\% são solteiros e 73,3\% não têm filhos. Ainda analisando os alunos ingressantes em cursos de pós-graduação lato sensu nesta IES, é possível concluir, através da análise do perfil profissional, que os alunos que procuram cursos de pós-graduação lato sensu almejam ascensão profissional.

Dentre as implicações acadêmicas dos resultados obtidos, podem-se destacar que a maioria dos estudos realizados sobre fatores de atração abordam alunos que já frequentam uma IES, e isso se deve à facilidade de abordagem para aplicação do questionário. A pesquisa com o público recém matriculado não é tão comum. Trata-se de uma contribuição relevante para as pesquisas na área do marketing educacional no que diz respeito às expectativas dos alunos ingressantes. É importante ressaltar ainda que os estudos anteriores sobre os cursos de pós-graduação lato sensu abordaram apenas uma área do conhecimento (gestão) e esse trabalho trouxe uma nova contribuição, pois analisou as expectativas dos alunos entrantes nas áreas de gestão, direito e saúde. 
Os resultados deste estudo podem fornecer, ainda, informações fundamentais para ações a serem produzidas nas IES. Dentre as implicações práticas, pode-se destacar a análise do perfil sócio demográfico e profissional do aluno recém matriculado. Tais informações podem antecipar à gestão das IES informações importantes para o marketing educacional. Por fim, por meio da análise dos atributos mais relevantes para escolha por cursos de pós-graduação lato sensu, os gestores podem direcionar suas ações de marketing.

No tocante às limitações do trabalho, cabe ressaltar que a pesquisa foi feita em uma IES da região da grande Vitória, no ES. Portanto, não podem ser feitas generalizações a respeito dos atributos de escolha por cursos de pós-graduação lato sensu para todas as IES. Sendo assim, recomenda-se a continuidade do estudo aqui iniciado para que os fatores determinantes sejam passíveis de generalização.

Para próximos estudos sugere-se que seja realizada uma abordagem onde as análises dos alunos dos cursos de pós-graduação lato sensu em gestão, direito e saúde sejam separadas. A amostra pode ser dividida também por faixa etária, formação e características de função profissional dos alunos. Desta forma, será possível verificar se existem diferenças relevantes entre as expectativas dos alunos entrantes de áreas e características distintas. Sugere-se ainda, que sejam feitas análises entre esses mesmos alunos no momento da conclusão do curso de pós-graduação. Pode ser feito um estudo à respeito de lealdade, satisfação e a influência dos atributos encontrados na presente pesquisa.

\section{REFERÊNCIAS}

AKAREEM, H. S.; HOSSAIN, S., S. Perception of education quality in private universities of Bangladesh: a study from students' perspective, Journal of Marketing for Higher

Education, v.1, p. 11-33, 2012.

ALFINITO, S.; GRANEMANN, S. R. Escolha de uma IES em função da utilidade do usuário potencial: o estudante. In: rocha, c. h.; granemann, s. r. (Org.). Gestão de instituições privadas de ensino superior. São Paulo: Atlas, 2003. p. 93-103.

ALVES, H.; MAINARDES, E. W.; RAPOSO, M. O marketing no ensino superior: comparativo Brasil-Portugal. Revista de Administração FACES - Jornal Belo Horizonte, v. 10, n. 4, p. 35-64, set./dez. 2010. Disponível em:

$<$ http://www.fumec.br/revistas/index.php/facesp/article/view/190/187/>. Acesso em: 03 jul. 2012.

BENNETT, R., ALI-CHOUDHURY, R. Prospective students' perceptions of University Brands: an empirical study. Journal of Marketing for Higher Education, v.19, n. 1, p.85107, jun. 2009. 


\section{ESCOLHA DE CURSOS DE PÓS-GRADUAÇÃO LATO SENSU E OS SEUS FATORES DETERMINANTES \\ DOI: http://dx.doi.org/10.5007/1983-4535.2015v8n3p219}

BERGAMO, F. V. et al. De prospect a aluno: fatores influenciadores da escolha de uma instituição de ensino superior. BASE - Revista de Administração e Contabilidade da Unisinos, Porto Alegre, v. 7, n. 3, p. 182-193, set. 2010.

CARVALHO, BaiardGuggi; BERBEL, Maurício Costa. Marketing educacional: como manter e conquistar mais alunos. 2. ed. São Paulo: Alabama, 2001.

COBRA, M.; BRAGA, R. Marketing educacional. 2. ed. São Paulo: Cobra Editora e Hopper Editora, 2004.

GIOTTO, O. T., GOMES, G., DOMINGUES, M. J. C. S. Educação continuada em administração: uma visão dos alunos concluintes em duas IES do sul do Brasil. In: CONGRESSO VIRTUAL BRASILEIRO DE ADMINISTRAÇÃO, 7., 2007, São Paulo (SP). Anais...São Paulo: Convibra, 2007.

HAIR JUNIOR, J. F., MONEY, A.; BABIN, B.; SAMOUEL, P. Fundamentos e métodos de pesquisa em administração. Porto Alegre: Bookman, 2005.

HOLANDA JUNIOR, A.; FARIAS, I. Q.; GOMES, D. M. de O. A. O valor do cliente como elemento de marketing para instituições de ensino superior. BASE - Revista de Administração e Contabilidade da Unisinos, v. 3, n. 2, p. 102-111, maio/ago. 2006.

INEP. Instituto Nacional de Estudos e Pesquisas Educacionais Anísio Teixeira.Resumo técnico do censo da educação superior: 2008 . .

KOTLER, P.; FOX, K. Strategic marketing for educationalinstitutions. 2. ed. EnglewoodCliffs (NJ): Prentice Hall, 1995.

LEITE, A. C. T.; LIMA, C. A. Técnicas e habilidades: educação continuada para a formação do administrador atual. In: ENCONTRO NACIONAL DA ASSOCIAÇÃO NACIONAL DOS CURSOS DE GRADUAÇÃO EM ADMINISTRAÇÃO (ENANGRAD), 8., 1997, Rio de Janeiro (RJ). Anais... Rio de Janeiro: ANGRAD, 1997.

LITTEN, L. Marketing higher education: benefits and risks for the American academic system. Journal of Higher Education, [S.1.], v. 51, n. 1, p. 40-59, 1980.

MAINARDES, E. W. Atração e retenção de alunos em cursos de graduação em administração das instituições particulares de ensino superior de Joinville/SC. 2007. Dissertação (Mestrado) - Programa de Pós-Graduação da Universidade Regional de Blumenau, Blumenau (SC), 2007.

MAINARDES, E. W., DOMINGUES, M. J. C. de S. Atração de alunos para a graduação em administração: estudo multicaso sobre os motivos de escolha de um curso superior no norte catarinense. Revista CESUMAR. , v.16, p.263 - 292, 2011.

MAINARDES, E. W., ALVES, H., DOMINGUES, M. J. C. de S. Cursos de administração em instituições privadas de ensino superior: estudo sobre os fatores de atração de estudantes em Joinville, SC. RGO. Revista Gestão Organizacional (Online), v.4, p.94 - 114, 2011. 


\section{ESCOLHA DE CURSOS DE PÓS-GRADUAÇÃO LATO SENSU E OS SEUS FATORES DETERMINANTES \\ DOI: http://dx.doi.org/10.5007/1983-4535.2015v8n3p219}

MAINARDES, E. W., DESCHAMPS, M., DOMINGUES, M. J. C. de S. O que atrai alunos para cursos de graduação em administração? In: VI Colóquio Internacional sobre Gestão Universitária na Amércia do Sul, 2006, Blumenau. VI Colóquio Internacional sobre Gestão Universitária na Amércia do Sul. , 2006.

MANTOVANI, N. O. Avaliação do ensino de administração na perspectiva de egressos e dirigentes de empresas. In: ENCONTRO NACIONAL DE CURSOS DE GRADUAÇÃO EM ADMINISTRAÇÃO, 6., 1995, Natal (RN). Anais... Natal: ANGRAD, 1995.

MELO, S.L; Borges, L.O. A transição da universidade ao mercado de trabalho na ótica do jovem. Psicologia Ciência e Profissão, v. 27, n. 3, p. 376-395, 2007.

MENEGHELLI, P. J. M. Atributos de atração para cursos superiores: um estudo com alunos ingressantes. 2011. Dissertação (Mestrado) - Programa de Pós-Graduação do Centro de Ciências Sociais Aplicadas da Universidade Regional de Blumenau, Blumenau (SC), 2011.

MEYER JUNIOR, V. Novo contexto e as habilidade do administrador universitário. In: MEYER JUNIOR, V.; MURPHY, J. P. (Org.). Dinossauros, gazelas \& tigres: novas abordagens da administração universitária: um diálogo Brasil-EUA. 2. ed. Florianópolis: Insular, 2003. p. 173-192.

MONTEIRO, L. A. S. A pós-graduação lato sensu em administração no Brasil: um estudo de caso. In: COLÓQUIO INTERNACIONAL SOBRE GESTÃO UNIVERSITÁRIA NA AMÉRICA DO SUL, 8., 2008, Assunção (Paraguai). Anais... Assunção: UFSC, 2008.

MUND, A. L.; DURIEUX, F.; TONTINI, G. A influência do marketing na opção do aluno pela Universidade Regional de Blumenau. In: CONGRESSO BRASILEIRO DE CIÊNCIAS DA COMPUTAÇÃO, 24., 2001, Campo Grande (MS). Anais... Campo Grande: SBC, 2001.

PAMPALONI, A. M. The influence of organizational image on college selection: what students seek in institutions of higher education, Journal of Marketing for Higher Education, v.1, p. 19-48, 2010.

PIÑOL, S. T. Janela do cliente-aluno nos cursos de pós-graduação. In: COLÓQUIO INTERNACIONAL SOBRE GESTÃO UNIVERSITÁRIA NA AMÉRICA DO SUL, 4, 2004b, Florianópolis (SC). Anais... Florianópolis: UFSC, 2004.

SCARPIN, M. R. S.; DOMINGUES, M. J. C. S.; SCARPIN, J. E. Fatores de atração como diferencial competitivo nos cursos de pós-graduação lato sensu. In: SEMINÁRIOS EM ADMINISTRAÇÃO (SEMEAD), 13., 2010, Rio de Janeiro. Anais... Rio de Janeiro: UFRJ, 2010.

SCARPIN, M. R. S; SCHARF, E. R.; FERNANDES, J. O valor da marca na escolha do curso de pós-graduação lato sensu de uma IES do vale do Itajaí - SC. In: SEMINÁRIOS EM ADMINISTRAÇÃO (SEMEAD), 14., 2011, São Paulo (SP). Anais... São Paulo: USP, 2011.

SILVA, Roberta D. de O. Fazer ver e crer: valores de educação na publicidade e propaganda escolar?. In: CONGRESSO BRASILEIRO DE CIÊNCIAS DA COMUNICAÇÃO, 28, 2005, Rio de Janeiro. Anais... Rio de Janeiro, XXVIII Congresso Brasileiro de Ciências da Comunicação, 2005. 
SIRVANCI, M. Are the students the true customers of higher education? Quality Progress, v. 29, n. 10, p. 99-102, out. 1996.

THIES, R. B.; BIANCHI, R. C. A visão dos formandos em administração e dos empresários com relação ao mercado de trabalho. In: CONGRESSO VIRTUAL BRASILEIRO DE ADMINISTRAÇÃO, 2., 2005, São Paulo (SP). Anais... São Paulo: CONVIBRA, 2005.

TIBOLA, J. T. Antecedentes da lealdade e da permanência de alunos em uma instituição de ensino superior. 2010. Dissertação (Mestrado) - Programa de Pós-Graduação do Centro de Ciências Sociais Aplicadas da Universidade Regional de Blumenau, Blumenau (SC), 2010. 